\title{
Warthin Tumor: A Common, Benign Tumor Presenting as a Highly Suspicious Mass
}

\author{
Robert L. Hatch, MD, and Sejal Shah, BS
}

Patients often present to family physicians complaining of swellings or "knots" in their neck. Many represent benign lymphadenopathy. However, especially in smokers and the elderly, this may be the initial presentation of life-threatening disease. We present a case of a worrisome neck mass in an older ex-smoker that proved to be a benign Warthin tumor. Although this is the second most common benign tumor of the parotid, ${ }^{1}$ neither we nor our colleagues were familiar with it. Furthermore, we could find no mention of it in the family medicine literature. The following case illustrates many of the features typical of Warthin tumor. This interesting tumor is the only benign neoplasm associated with smoking. ${ }^{1}$ A better understanding of this tumor could help family physicians maintain a broader initial differential diagnosis in such cases and improve their understanding of the diagnostic approach to masses in this area.

\section{Case Report}

A 78-year-old white man with a history of melanoma of the ear presented for evaluation of a neck mass. He first noted the mass 2 years earlier. He reported that it was larger at first but shrank slightly after a course of oral antibiotics. Since that time, its size had not changed. The mass was completely asymptomatic. Review of systems revealed no hoarseness, dysphagia, or weight loss. Twelve years earlier, he had been diagnosed with level 1 Clark melanoma of the pinna of the left ear. He underwent prompt excision, and no recurrence had been noted on follow-up. Past medical history was also positive for multiple medical problems, including recurrent transient ischemic attacks, myocardial infarction, coronary artery bypass grafting, conges-

Submitted, revised, 18 March 2005.

From the Department of Community Health and Family Medicine, University of Florida, Gainesville.

Conflict of interest: none declared.

Corresponding author: Robert L. Hatch, MD, CHFM, Box 100222, University of Florida, Gainesville, FL 32610 (email: hatch@dean.med.ufl.edu). tive heart failure, cardiac pacemaker, and automatic implanted cardiac defibrillator (AICD) for ventricular tachycardia. He was taking multiple medications, including warfarin. Social history was positive for 75 pack-years of smoking, although the patient quit 17 years before the visit. Physical examination revealed a $2 \times 2-\mathrm{cm}$ nontender mass below the angle of the left jaw. The consistency of the mass was slightly softer than a typical lymph node. The mass was mobile and did not seem to be attached to either the skin or underlying structures. No other masses were noted in the head and neck. No adenopathy was noted in the groin, axilla, or extremities. Skin revealed no evidence of melanoma recurrence.

Because of the patient's pacemaker and AICD, magnetic resonance imaging (MRI) was not performed. Soft tissue computed tomography (CT) of the neck (without contrast because of prior anaphylaxis from contrast) revealed a $2-\mathrm{cm}$ mass at the lower pole of the parotid (Figure 1). The mass was dense with lower central density and well defined margins. Given the patient's history, the CT was read as highly suspicious for nodal metastasis of melanoma. Warfarin was temporarily suspended, and fine-needle biopsy was performed without complication. Biopsy revealed glandular epithelial cells in a lymphoid stroma, the characteristic histology of a benign Warthin tumor (see Figure 2). The patient recovered from the procedure uneventfully. After discussion of treatment options, the patient chose no further treatment. Six months after biopsy, the mass remained asymptomatic and essentially unchanged.

\section{Discussion}

Warthin tumor, also known as papillary cystadenoma lymphomatosum, ${ }^{2}$ is a fairly common tumor. It makes up $14 \%$ to $30 \%$ of parotid tumors ${ }^{1}$ and is well known among otolaryngologists. The case described above is typical of Warthin tumor. The patient's age, smoking history, lack of symptoms, and delay in presentation are all typical. Warthin 


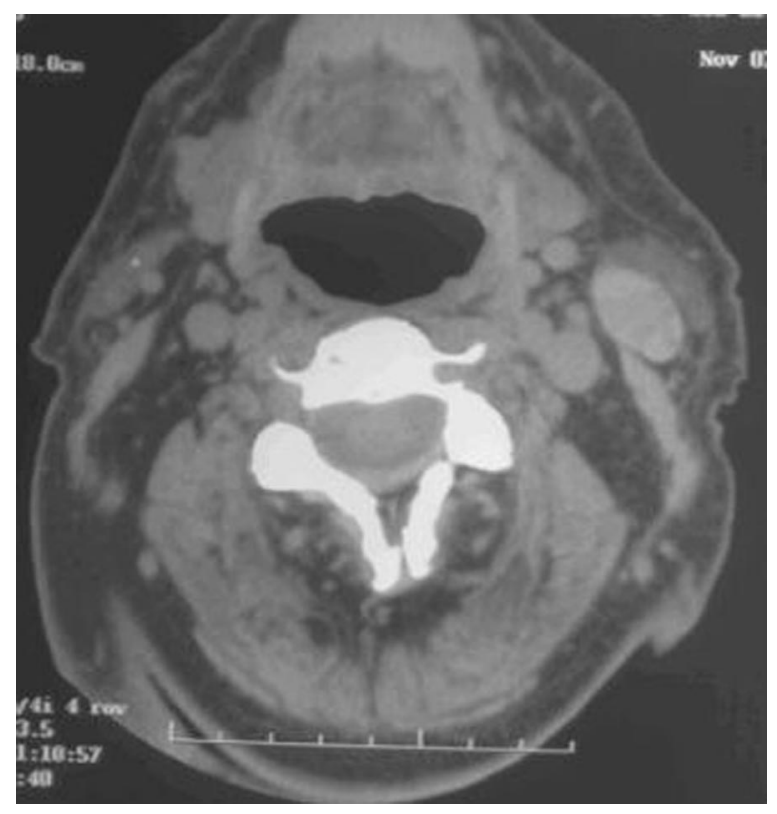

Figure 1. Unenhanced CT image of Warthin tumor of the parotid gland (arrow). Note the well defined margins of the tumor and the lower central density.

tumor almost always occurs in older adults. In men, the peak incidence is in the 7 th decade, whereas in women it is in the 6 th decade. ${ }^{3}$ Because the tumor

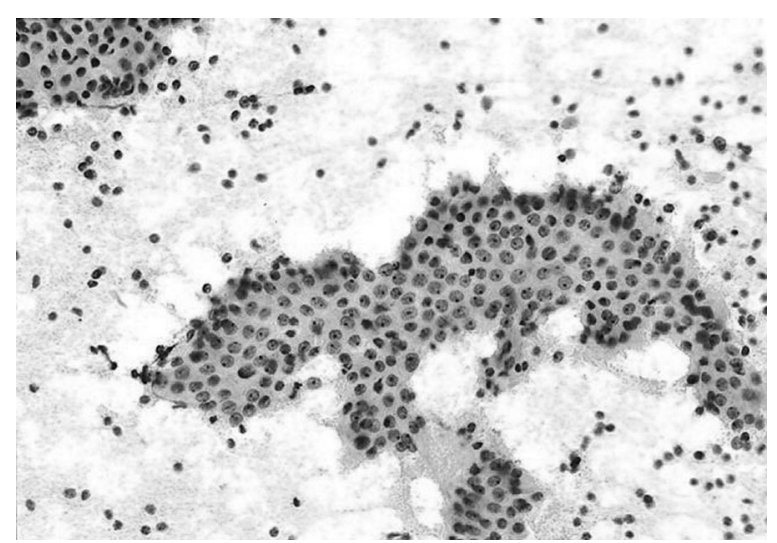

Figure 2. Warthin tumor of the parotid gland: Fineneedle aspiration reveals the classic cytomorphologic appearance of the tumor with flat, monolayered (papillary-like) sheets of oncocytic cells; background lymphocytes; and cystic debris (Papanicolaou stain; original magnification, $200 \times$ ). (Reproduced from Parwani AV, Ali SZ. Diagnostic accuracy and pitfalls in the fine-needle aspiration interpretation of Warthin's tumor. Cancer Cytopathology 2003;99:166-71. Copyright $\odot 2003$ American Cancer Society. Used with permission.) occasionally occurs in young patients, most studies have found an average age at diagnosis in the early 60 's. ${ }^{1}$ Smokers have 8 times the risk of developing Warthin tumor ${ }^{4}$. This is hypothesized to be caused by retrograde flow of substances in tobacco smoke into salivary ducts or by excretion of substances from smoke into the ducts. ${ }^{5}$ The tumor is asymptomatic in $90 \%$ of patients. ${ }^{2}$ Occasionally, patients may note pain, pressure, or rapid growth of the mass. If the mass ruptures, the contents can cause inflammation and acute parotitis. ${ }^{2}$ Patients often delay seeking medical care for Warthin tumor. In a study of 278 cases, the average time between appearance of the mass and diagnosis was 21 months. $^{3}$ The asymptomatic nature of this tumor probably explains much of this delay.

Although Warthin tumor can occur elsewhere, it is most commonly found within the parotid gland. In one series, essentially all were located in the parotid. ${ }^{3}$ Another series found $90 \%$ within the parotid, $7.6 \%$ in cervical lymph nodes, and $2.3 \%$ in the submandibular gland. ${ }^{1}$ Before 1982, Warthin's tumor made up $6 \%$ to $16 \%$ of parotid tumors. ${ }^{1}$ In the past decade, the incidence of the tumor has doubled. ${ }^{1}$ This is not surprising, because the population is aging, and many older patients have a history of smoking. At first, Warthin tumor was seen primarily in white men. ${ }^{1}$ It is becoming more prevalent in both African Americans and women. Indeed, the gender ratio now approaches $1: 1{ }^{1}$ The increased prevalence in African Americans and women is thought to be caused by increased smoking among these groups.

On standard MRI, Warthin tumor has certain characteristic features, including multifocal lesions and well defined margins ${ }^{6}$. However, these features are not diagnostic. ${ }^{6}$ Several recent reports have examined the value of dynamic MRI in differentiating Warthin tumor from malignant neoplasms of the parotid. Dynamic MRI is reported to have a high specificity (91\%) and sensitivity (91\%) for Warthin tumor. ${ }^{7}$ These investigators correctly identified all Warthin tumors as benign. However, their diagnostic scheme was not able to differentiate all malignant parotid tumors from benign ones. With further study and advancement, MRI technology may one day be able to establish the diagnosis in such masses.

A definitive diagnosis therefore requires a tissue sample. In the past, most specimens were excised and the diagnosis was established after excision. 
Fine-needle aspiration (FNA) has played a larger role in recent years. In general, FNA has good overall accuracy for diagnosing salivary gland neoplasms ( $87 \%$ to $97 \%),{ }^{8}$ and it has been widely used for the diagnosis of Warthin tumors. It is generally well tolerated, although inflammation and development of a sialocutaneous fistula have occurred after FNA of Warthin tumor. ${ }^{2}$ A positive cytological diagnosis requires the distinct histologic elements of oncocytic epithelial cells (ie, acidophilic and granular) in a lymphoid stroma (Figure 2). ${ }^{9}$ One important limitation should be kept in mind when using FNA to assess a possible Warthin tumor. Whereas it is rare for a malignant lesion to be misclassified as Warthin tumor, as many as $26 \%$ of Warthin tumors may be misclassified as malignant on FNA. ${ }^{10}$

Optimal treatment of Warthin tumor remains somewhat controversial. According to Batsakis, who has written extensively about Warthin tumor, they are "generally regarded as among the most innocuous of salivary glad tumors." Malignant transformation is very rare and constitutes only $0.3 \%$ of all Warthin tumors. ${ }^{9}$ Despite this, Yoo et $\mathrm{al}^{1}$ recommend superficial parotidectomy but recognize that either local excision or simple observation would be appropriate in selected cases. Their preference is based in part on a recurrence rate of $1.8 \%$ after local excision. ${ }^{11}$ These may not have represented true recurrences, however, because patients often have multiple Warthin tumors. Texts that are more recent do little to resolve the treatment controversy. One author states that "treatment requires complete excision of the affected portion of the gland with uninvolved margins."12 Another states that fine-needle biopsy results allow "for a discussion of non-surgical options in a patient with a benign Warthin's tumor."13

Given this controversy, consultation with an otolaryngologist and individualization of treatment is recommended. In the case described above, the patient chose observation alone, in part because of his multiple medical problems and higher operative risk.

\section{References}

1. Yoo GH, Eisele DW, Askin FB, Driben JS, Johns ME. Warthin's tumor: A 40-year experience at The Johns Hopkins Hospital. Laryngoscope 1994;104: 799-803.

2. Batsakis JG, El-Naggar AK. Warthin's tumor. Ann Otol Rhinol Laryngol 1990;99:588-91.

3. Eveson J, Cawson R. Warthin's tumor (cystadenolymphoma) of salivary glands. A clinicopathologic investigation of 278 cases. Oral Surg Oral Med Oral Pathol 1986;61:256-2.

4. Kotwall C. Smoking as an etiologic factor in the development of Warthin's tumor of the parotid gland. Am J Surg 1992;164:646-7.

5. Ebbs SR, Webb AJ. Adenolymphoma of the parotid: aetiology, diagnosis and treatment. Br J Surg 1986; 73:627-30.

6. Minami M, Tanioka $\mathrm{H}$, Oyama $\mathrm{K}$, et al. Warthin tumor of the parotid gland: MR-pathologic correlation. AJNR Am J Neuroradiol 1993;14:209-14.

7. Yabuuchi H, Fukuya T, Tajima T, Hachitanda Y, Tomita K, Koga M. Salivary gland tumors: diagnostic value of gadolinium-enhanced dynamic MR imaging with histopathologic correlation. Radiology 2003;226:345-54.

8. Stewart CJ, MacKenzie K, McGarry GW, Mowat A. Fine-needle aspiration cytology of salivary gland: a review of 341 cases. Diagn Cytopathol 2000;22:13946.

9. Batsakis JG. Carcinoma ex papillary cystadenoma lymphomatosum malignant Warthin's tumor. Ann Otol Rhinol Laryngol 1987;96:234-5.

10. Parwani AV, Ali SZ. Diagnostic accuracy and pitfalls in the fine-needle aspiration interpretation of Warthin's tumor. Cancer Cytopathology 2003;99:16671.

11. Heller KS, Attie JN. Treatment of Warthin's tumor by enucleation. Am J Surg 1988;156:294-6.

12. Butt FY. Benign diseases of the salivary glands. In: Lalwani AK, ed. Current diagnosis and treatment in otolaryngology: head \& neck surgery. New York: McGraw-Hill, 2002. p. 307-24.

13. Randolph QW. Salivary gland disorders. In: Wilson WR, Nadol JB, Randolph GW, eds. The clinical handbook of ear, nose and throat disorders. New York: Parthenon Publishing; 2004. p. 377-394. 